\title{
Effects of Environment on Gestation Ratio of Sheep Embryo Transfer and its Control Measures
}

\author{
Shangxin Sun \\ Chaoyang Engineering Technical School, Chaoyang, Liaoning 122000, China \\ Tel: 86-421-661-0935_E-mail: ssx2912712@163.com
}

\begin{abstract}
There are a lot of factors affecting the gestation ratio of sheep embryo transfer, and among them, environmental condition is an important one. We should provide the transferred embryos a better environmental condition for their survival and growth to increase the gestation ratio of sheep embryo transfer.
\end{abstract}

Keywords: Environment, Sheep embryo transfer, Gestation ratio, Control

There are lots of factors affecting the continuous pregnancy after embryo transfer. Those factors are very complicated, including the physiological, endocrine, genetic, immunological and environmental factors. Currently, many experts and scholars classified all these factors into three aspects, namely embryo factors, receptors and other factors. However, according to our practical observation and investigation review, we drew a conclusion that environmental condition was one of the most paramount factors affecting the gestation ratio of embryo transfer.

\section{Effects of environmental factors on gestation ratio of sheep embryo transfer}

According to the theory of Livestock Environmental Health, the external environment refers to an integration of three elements, including the atmosphere, water, and soil, and it is the material basis for biological survival. The three elements have their physical, chemical and biological characteristics respectively(Wang, 1981, PP. 1-71). As for the livestock, the concept of environment was more extensive. Besides above-mentioned three elements, it contained animal husbandry, management, tuning and usage by human beings, as well as rangeland, pasture, housing and relationships within the livestock group, i.e., any environmental condition concerning the living and production of livestock belonged to the environment (Zhou, 1990, PP. 1-40). Above-mentioned environmental conditions could be called large environment for the implanted embryos. Embryos grew in the reproductive system organs of parent body; accordingly, parent body was called small environmental condition for the survival of embryos.

\subsection{Effect of large environmental factors on gestation ratio of embryo transfer}

\subsubsection{Effects of solar radiation on sheep reproductive physiology}

Sheep belong to short day animals, and usually only in certain seasons have seasonal polyestrus and reproduce. Illumination variation from short to long, or from long to short, could cause changes of endocrine activities through nervous system (Zhou, 1990, PP. 1-40). Practice showed that the gradual reduction of sunshine time played a cardinal role in the sheep seasonal polyestrus. Light acted on the hypothalamus and released neurohormones through different pathways, regulated the secretion of anterior pituitary gonadotropin through the pituitary portal blood flow, and thus indirectly controlled the produce of eggs and sex hormones in ovaries. After seasonal polyestrus, when sunshine time changed from short to long, the development of eggs and the produce of sex hormones would be inhibited in ovaries, and sheep stepped into reproductive physiological processes without estrus. As the solar radiation directly affected reproductive physiology of ewes, and thus had a direct or indirect impact on the gestation ratio of embryo transfers.

\subsubsection{Effects of temperature on animals and embryo physiology}

Firstly, effects of the ambient temperature on animals' reproductive biology and the development of embryo transferred. Tremendous experiments showed that in the abnormal cold winter, the estrous cycle of cattle tended to reduce, and egg production of hens decreased or ceased. When the temperature was appropriate in spring, the follicular development of mare progressed rapidly, and when confronting sudden cooling, follicular growth and development would be inhibited. Accordingly, temperature changes indeed interfered with the process of animal reproductive physiology.

High temperature could cause endocrine imbalance, especially the reduction of thyroid hormone secretion, and 
have negative effects on the fecundity of both males and females. When the ambient temperature was too high, reproductive function of receptor sheep would change, which could easily cause the weakness and death of embryos before or after implantation. If male sheep suffered from continuous high temperature in pregnancy period, primary newborn animals would get a smaller body size, lower viability and higher mortality.

According to the swine farrowing data of Iowa in USA, the critical period of effects of high temperature on conception rate and embryonic mortality was within 8 days after hybridization and 11-20 days after conception (Wang, 1981, PP. 1-71).

If temperature was too low and low temperature kept a long time, body temperature of livestock would reduce continuously, the central nervous system would be inhibited, and the nerve conduction would suffer from obstacle, which would result in lower reactivity of the body, reduction of metabolic rate, drop of the reproductive physiology of livestock, and thus depravation of embryos growth and viability.

Secondly, effects of ambient temperature of embryo inspection rooms and operating rooms on embryos. Temperature variation had a great impact on the survival of embryos. The temperature in embryo inspection rooms and operating rooms should be maintained at a certain scope, and suitable temperature should range from $20{ }^{\circ} \mathrm{C}$ to $25{ }^{\circ} \mathrm{C}$. If the room temperature reduced to below $15{ }^{\circ} \mathrm{C}$, the temperature condition would have a negative impact on the survival of the embryos, or even seriously acerbate the survival of embryos.

Consequently, too high or low ambient temperature that last a long time would seriously affect the animal's reproductive function and embryo growth and vitality.

\subsubsection{Effects of nutrition on reproductive function of dams and embryos.}

Ingredients and quality of nutrition, including feed and fodder, had long-term and gradual effects on the reproductive performance of ewes Nutritional deficiencies could cause no estrus, and long-term feed without enough heat, or in short of certain particular nutritional elements, would gradually result in complete no estrus. Nutritional deficiencies could lead to the barriers blocking the release of pituitary gonadotropin, or weak response of ovary to pituitary gonadotropin. Meanwhile, the nutritional status of the receptor would affect the survival of embryos in the body, the formation of organs and tissues after implantation, and the final birth and survival of lamb.

\subsubsection{Effects of operating room environmental sanitation level on embryos}

Air sanitation of the operating room and embryo inspection room was very significant for embryo transfers (Li, 2004, PP. 42-72). If more dust there, there would be more poisonous and harmful substances in the air, and that would cause pollution to utensils and equipments that would contact with embryos, and thus embryos got impairment. Appliances and equipment in contact with embryos, such as punching egg tube, shift egg tube, egg concentration utensil, petri dish, pipette, bacteria-reactor, driver egg needle and so on, should not be contaminated. Otherwise, it would result in the invasion of pathogens, or even embryonic death.

\subsection{Effects of small environment of donors and receptors on gestation ratio of sheep embryo transfer}

\subsubsection{Effects of endocrine and hormone on the reproductive physiology of donors and receptors.}

The reason that livestock became a harmonious unit was that they had an accurate and complete integrated organization. Endocrine system is one of the two integrated organizations in animal body. Many important physiological processes in body, such as estrous and pregnancy, were quite accurate and constant, and this could be attributed to the coordinate activities of various endocrine hormones. Some of the more important integrated domains have been replaced by nervous system in higher animals, however, in the overall coordination process they both were complementary, and had their own irreplaceable functions and powers respectively.

Hormones were products from every endocrine secretion. Hormones played a special significant role in many activities in bodies, including growth, differentiation, and reproduction, maintaining the harmony of internal and external environmental and other aspects. As for guaranteeing reproduction, incretion was closely associated with reproduction, from the generation and maturation of gametes, to the formation and pregnancy of zygotes, and then to infantile birth and lactation, and all those processes required the direct involvement of reproductive hormones.

Consequently, endocrine system and its hormones had an obvious effect on the reproductive physiology of donors and receptors, and were important factors of small environmental condition for embryo formation and living after implantation.

\subsubsection{Estrus synchronization and its effects}

Estrus was one of the most important and fundamental physiological phenomenon in female reproduction (Zuo, 
2004, PP. 34-35). In practice, in order to maximize reproductive rate, people attempted to induce estrus synchronization of dams using several hormones. By artificially regulating and controlling estrus rules, people obtained estrus and ovulation of dams in an expected time so as to designedly organize the hybridization. We called such livestock breeding technologies as estrus synchronization.

Embryo transfer required more estrus synchronization of donors and receptors. Therefore, during the process of transfer embryo, using estrus synchronization technology, the status of ovaries of the treated livestock were changed as expected, and their reproductive physiologies were relatively at a common level. Artificial estrus synchronization resulted in embryo implantation within the same environment and tremendous receptors susceptible to transfer.

Modern estrus synchronization technology had two approaches ( $\mathrm{Li}, 2004$, PP. 42-72). One was to apply progesterone on a swarm of dams to be treated, inhibit the growth and development of ovarian follicle and estrus, and induce the estrus synchronization after a certain period of drug withdrawal. The other was to dissolve corpus luteum using prostaglandins, reduce progesterone level, promote the release of pituitary gonadotropins, and trigger estrus. Although those two approaches applied two different hormones with different effects, they all resulted in the same consequence after treatment, namely estrus synchronization.

Embryo transfer relied on the estrus synchronization of donors and receptors seriously. Considerable practical experience showed that the survival rate of sheep transfer embryos could be larger as long as the starting time of estrus in both donors and receptors were closely synchronized, when other conditions were suitable. If the starting time of estrous between donors and receptors was closer, the receptor sheep would obtain a higher conception rate. If the estrus of donors and receptors was not synchronized, conception rate would decline; if the difference was more than $12 \mathrm{~h}$, conception rate of receptors would significantly decreased; if more than $48 \mathrm{~h}$, it would almost be no possibility for conception.

\subsubsection{Effects of embryo transfer sites}

Sheep embryo transfer site was an important factor of small environment of receptors. Receptor sheep were not pregnant before embryo transfer, but their reproductive organs including fallopian tubes, ovaries and uterus, continued reproductive physiological changes during different stages according to the specific procedures as follows: estrus-ovulation-fertilization-pregnancy, including the whole physiological changes in bodies. All those changes tried to get preparations for the pregnancy including the movement, implantation, differentiation, growth and development of embryos. Due to different function of each organ, there was a certain difference among their internal environment. If time difference was bigger, the internal environment in same sites would be different $(\mathrm{Li}$, 2004, PP. 42-72). Consequently, if the transfer sites were different, the physiological and biochemical environment of embryos would be distinct, and small environment of embryos would be seriously ruined in that way, which could threaten the survival of implanted embryos directly.

\subsubsection{Effects of receptor physiological status}

The physiological status of uterus and ovary in receptors had a great impact on transfer embryos, including before and after operation. If receptors had diseases in reproductive system, especially for the infection of uterus and fallopian tubes by bacteria, the survival and development of embryos would be influenced. If they have a history of abortion, or weaning time of postpartum ewes was not long enough, reproductive organs and their functions would not fully recover, which would also affect the survival rate of implanted embryos. Due to the decreased resistance after operation, dirty stables, damages to anatomical layers and muscle, the foreign body and hematoma of surgical sites, and blunt and rough operation of transplantation practice, all those abovementioned factors could lead to infection after operation. The infection would not only destroy the environmental conditions for implanted embryos, affect the efficiency of transfer embryos and cause embryo death, but also result in the lifetime infertility of receptor sheep.

\section{Approaches to improve gestation ratio of sheep embryo transfer}

Environmental factors, including large and small environment, especially for the receptor environment were the important condition directly affecting the gestation ratio of sheep embryo transfer. According to our practical observation and experience review of over 10,000 cases of sheep embryo transfer work for many years in our region, we offered the following suggestions as references for practical work.

\subsection{To provide the same or very similar large environmental conditions with donors and receptors}

\subsubsection{Consistency of the natural environment}

The natural environment includes solar radiation, temperature, altitude, latitude and longitude, and other natural 
and geographical conditions. If possible, receptor ewes should be centralized and offered the same natural environment with donors. Both donor and receptor ewes grew, developed and propagated at the same environmental condition, naturally obtained the same reproduction and physiological function, so as to create favorable donor and receptor conditions for smooth embryo transfer. As for the poor farms where the same conditions could not be afforded, both donors and receptors should be centralized as much as possible. Within 3 or 4 months before embryo transfer, receptors should be given the same or similar environmental conditions with donors in order to establish a good basis for embryo transfer work.

\subsubsection{Consistency of nutrition or feed and pasture.}

As embryo transfer demands that receptors should attain an excellent breeder body condition, their nutrition should be balanced, comprehensive and sufficient. During the period of transfer that receptor accepted, besides that fatness should attain the middle level or a higher level, their nutritional level should be in a more stable increasing phase. Even if the fatness of ewes was excellent, but if their nutritional level was in a decreasing trend, survival rate of embryos would decrease, which was the issues that more attention should be paid to in embryo transfer.

\subsubsection{Consistency of feeding management}

In embryo transfer work, feeding and management manner also interfered with the reproductive physiological processes of donors and receptors. Conditional stock farms should raise livestock mainly using barn feeding, in combination with enough sports as grazing for some time. Some farms maybe completely feed on barn feeding, and had adequate sports venues. Various types of breeding sheep should be feed separately as groups. Natural mating should be strictly prohibited, and the ram barns should be strictly separated from the other sheep and ambient environment. Livestock farms had solid walls to prevent the access of other animals and unrelated person. In either way, it could be a good way for donors and receptors to exercise at the same feeding and management manner, which was quite essential for both donors and receptors to achieve the same or similar reproductive physiological conditions.

\subsubsection{Stick to aseptic technique and strict surgical procedures.}

Sticking to aseptic technique and strict surgical procedures is one of the important working contents to improve the large environment conditions of embryo transfer. Embryo transfer should be performed in the operating room with embryo inspection room inside, and the two rooms should be completely disinfected to achieve sterile room standards. Surgical equipment and instruments should be strictly sterilized, and it could be better if there were sterile incubator or super clean benches in operating rooms. Preparation room should be located outside the operating room, and strictly sterilized. Its function was to capture the sheep, wet wool, reduce dust, reduce pollution and prevent bacterial infection. Hats, shoes, boots of the operators and assistants as well as themselves should be strictly sterilized. Aseptic level in operating rooms and inspection embryo rooms should be controlled in order to prevent damages to embryos caused by contamination.

\subsection{Create good small environment for embryo survival, and promote gestation ratio of embryo transfer}

The small environment of embryo survival is receptor ewes. On the premise of making good large improvements, all kinds of work after or before receptor ewe embryo transfer should be well done carefully in order to ensure good survival conditions after implantation and thus improve the pregnancy rates.

\subsubsection{Consistency of the reproductive physiological age of donors and receptors}

One of the basic principles for us to selecting receptor ewes was to choose those with strong reproductive physiological function and multiparity. Based on this, it could be better that the reproductive physiological age of receptors were identical or very similar to that of donors. Certainly, it was the most ideal situation. It was very difficult to achieve such an ideal situation in our production practice. Accordingly, we selected the ewes with strong reproductive physiological function and multiparity as receptors. If their reproductive physiological ages were same or extremely similar, the reproductive physiology of both donors and receptors were identical. They were susceptible to attain the total estrus synchronization and their implanted embryos were also extremely likely to survive.

\subsubsection{Same or similar birth order and embryo transfer times}

The birth order of donors and receptors could be also used as an important metric to measure its reproductive physiological age. Same or similar birth order made their reproductive physiological age similar, hence their reproductive physiological function was similar too, which would provide suitable environmental conditions for embryo transfer quite easily. As far as embryo transfer times were concerned, due to the application of 
exogenous hormones on both donors and receptors, after repeated implantation, the reproductive physiology of receptors was bound to be affected in different degrees, and its function was attenuated. Therefore, initiating from controlling the small environment of implanted embryo receptors, selecting ewes with same embryo transfer times as receptors was relatively better. The reproductive physiologies of receptors and donors were easily synchronous, and the small environment of receptors would be easily controlled. For example, for the old donors, young and strong receptors with vigorous resistance should be selected.

\subsubsection{Consistency of application of exogenous sex hormones and regulation of the repeated usage}

There were many factors to affect the artificially controlled reproduction of dams, among which one of the important points was the characters of the hormones and their using methods, including type, batch, effectiveness, dosage, proportion, usage program, and producer. Consistency of application of exogenous sex hormones and regulation of the repeated usage should be followed. For practical application of hormones, appraisal test should be performed on several dams in order to observe the reactions and validate the hormone titer. According to the test results, batches of dams would be treated properly. The applied drug should be the same type and batch. If there were receptors and donors with multiple embryo transfers, due to multiple drug application, they could not only enhance resistance to drugs, but also their reproductive organ function degraded. Therefore, the dosage should be adjusted in order to achieve satisfactory results. It could be better that receptors and donors after multiple artificial-controlled reproductions were artificially controlled to reproduce in same large environment, which could not only control and regulate the dosage, but also be easy to create the same small environment for both donors and receptors.

In addition, in order to improve the function of reproductive organs, receptors with implanted embryos after surgery should be intramuscularly injected with progesterone to enhance assisted reproductive hormone levels. This would provide more suitable receptor environment for implanted embryos, and improve their survival rate.

\subsubsection{Consistency of transfer sites}

The sites that embryos were transfer to in the reproductive organs of receptors should be the same with donors, which was a very strict principle. According to where the original embryo was located in the body of donors and age of embryos, implanted sites were determined. Namely embryos out from fallopian tube should be implanted into the fallopian tube, so it was the same with uterine horn. Accordingly, the requirements of embryos for the small environment of both donors and receptors were extremely stringent.

\subsubsection{Preoperative disease control of receptors and prevention of postoperative infection}

Before embryo transfer, due to the strict selection of sheep receptors, receptors with good reproductive performance could be guaranteed, without reproductive system diseases and infectious disease. The ewes with certified quarantine, physical health, slightly large body size and multiparity were selected as receptors, and they would need no treatment. Preoperative prevention mainly was to place sponges or CIDR which should be strictly sterilized in order to prevent vaginitis that resulted in the decline of embryonic survival rate. The prevention of postoperative infection was also very important, and could directly influence the effects of embryo transfers. Consequently, the postoperative prevention with appropriate antibiotics was essential. The treatment with antibiotics mainly contained intramuscular injection, intravenous injection, and intraperitoneal injection. Considerable types of antibiotics could be applied, such as penicillin, streptomycin, and drug combination, 2 times each day, continuous for 2-3 days.

In conclusion, the environmental factors played a critical role in affecting the gestation ratio of embryo transfer. We attempted to study the relationship between embryos and the large external environment or the small environment of donors and receptors. Better, completely similar if possible, conditions of large environment and small environment of donors and receptors should be artificially created for embryo transfer to guarantee the survival of the implanted embryos, improve the embryo transfer, and accomplish sheep embryo transfer works with high qualities and quantities.

\section{References}

Li, Y.C. (2004). Sheep embryo transfer practical skills. Golden Shield Press, 12, 42-72.

Wang, Q.G. (1981). Livestock environmental health. Agriculture Press, 4(1):1-71.

Zhou, D.K. (1990). Livestock environmental health. Agriculture Press, 5(1):1-40.

Zuo, B.Y., \& Chen, J.Z. (2004). Test results of different ovarian stimulation schemes in Xuhuai white goats. China Animal Husbandry \& Veterinary Medicine, 32 (3): 34-35. 